\title{
Erratum To: Advances in Applications of Industrial Biomaterials
}

Eva Pellicer, Danilo Nikolic, Jordi Sort, Maria Dolors Baró, Fatima Zivic, Nenad Grujovic, Radoslav Grujic and Svetlana Pelemis

\section{Erratum to:}

E. Pellicer et al. (eds.), Advances in Applications of Industrial Biomaterials, https://doi.org/10.1007/978-3-319-62767-0

In the original version of the book, the belated correction to change the 4th volume editor's name from "Maria Baró" to "Maria Dolors Baró" in Frontmatter and cover page has to be incorporated. The erratum book has been updated with the change.

The updated online version of this book can be found at https://doi.org/10.1007/978-3-319-62767-0 